\title{
Do the dimensions of the hard palate have a relationship with the volumes of the upper airways and maxillary sinuses? A CBCT study
}

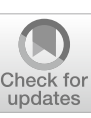

Murilo Miranda-Viana ${ }^{1 *}$, Deborah Queiroz Freitas ${ }^{1}$, Alessiana Helena Machado ${ }^{1}$, Amanda Farias Gomes ${ }^{1}$ and Yuri Nejaim²

\begin{abstract}
Background: As the hard palate is a central structure of the skull, and its close relationship with the nasal cavity, oral cavity, and maxillary sinuses, it would be of interest to study if there is a relationship between this bone and other structures of the stomatognathic system. Thus, this study aimed to assess the dimensions of the hard palate and associate them with sex, and skeletal and breathing patterns. Also, to investigate if there is a relationship between these dimensions and the volumes of the upper airways and maxillary sinuses.

Methods: Two hundred and ninety-eight CBCT scans of patients were classified according to sex, and skeletal and breathing patterns. Then, the linear dimensions of width and height of the hard palate at the regions of the first premolars and first molars, and the volumes of the upper airways and maxillary sinuses were measured using the CS 3D Imaging and ITK-SNAP software, respectively. Data were submitted to multi-way analysis of variance and linear regression, with a significance level of $5 \%(a=0.05)$.

Results: Sex and facial type influenced the hard palate dimensions $(p<0.05)$. Males had greater width and height of the hard palate than females $(p<0.0001)$. It was observed greater width for brachycephalics at the first premolars region $(p=0.0032)$, and greater height for dolichocephalics at the first premolars $(p=0.0154)$ and first molars $(p=0.0038)$ regions. Skeletal malocclusion and breathing pattern did not influence the measurements of the hard palate $(p>0.05)$. There was a significant relationship between the width and height of the hard palate at the premolar's region and the total volume of the upper airways $(p=0.018$, and $p=0.038)$, and between both dimensions of the hard palate at the molar's region and the total volume of the maxillary sinuses $(p<0.0001)$.

Conclusions: The hard palate dimensions are influenced by sex and facial type, but not by skeletal malocclusion or breathing pattern. Also, there is an association between these dimensions and the volumes of the upper airways and maxillary sinuses.
\end{abstract}

Keywords: Cone-beam computed tomography, Hard palate, Maxillary sinus, Pharynx

\footnotetext{
*Correspondence: muriloomiranda@gmail.com

${ }^{1}$ Department of Oral Diagnosis - Oral Radiology Area, Piracicaba Dental

School, University of Campinas, Piracicaba, SP, Brazil

Full list of author information is available at the end of the article
}

\section{Background}

The hard palate is located at the lower third of the skull, and is composed by the palatal process of the maxilla and the horizontal plate of the palatal bone. This bone plays an important role in the craniofacial complex, as it is involved in orofacial functions such as chewing, swallowing, phonation and breathing [1]. 
Studies in the literature have reported that the morphology of the hard palate may be influenced by the muscular tension, which can vary with the facial type, and skeletal and breathing pattern of the individual [2-4]. The characteristic stretched and weaker musculature of dolichofacial and mouth-breathing patients, for example, exert less pressure on the bone tissues, leading to possible alterations in the development of the craniofacial complex, including the maxilla and the hard palate [4-6]. Given the central location of the hard palate and its close relationship with the nasal cavity, oral cavity, and maxillary sinuses, it is possible that changes in its morphology may also affect other structures of the stomatognathic system [7-9].

Only few researches [6-8] had the hard palate as an object of study, and most of them were performed with small samples and in pediatric patients-a period in which the bones and integumentary tissues are not yet fully developed-and/or in syndromic patients, restricting the obtained conclusions to these specific groups. Additionally, all these studies were conducted in bidimensional radiographs, which present overlapping images and magnification/distortion of structures, or in plaster models, which may present distortions inherent to the molding process or to the plaster itself. Therefore, studies on the hard palate, using modalities of exams with higher accuracy in the evaluation of the craniofacial structures, such as cone beam computed tomography (CBCT), are of interest [10]. To the best of the authors knowledge, there are no studies on the evaluation of a possible relationship between the hard palate and the volumes of the maxillary sinuses and/or upper airways.

Therefore, the aim in this study was to assess the dimensions the hard palate and associate them with sex, and skeletal and breathing patterns. Also, to investigate if there is a relationship between these dimensions and the volumes of the upper airways and maxillary sinuses.

\section{Methods}

This observational, cross-sectional, and retrospective research was initiated after approval by the local institutional review board (IRB) under the protocol number: 3.491.476.

\section{Sample selection}

Initially, 340 cone beam computed tomography (CBCT) scans with extended FOV (field of view) and pertaining to the database of oral radiology clinic of Piracicaba Dental School (São Paulo, Brazil). The images were obtained prior to the present research (January 2014 to December 2016), and for clinical reasons not related to it. Images of patients 18 years old or older, of both sexes, with all teeth present (third molars were not required), were included in the sample. CBCT scans of patients with history of trauma or orthognathic surgery, presence of bone fracture, syndromes, bone exostoses, pathological lesions, or cleft lip/palate, as well as scans with presence of artifacts impairing the evaluation of the anatomical structures of the head and neck, were excluded from the sample.

Hence, after employing the exclusion and inclusion criteria, the final sample for the morphometric assessment of the hard palate, and the volumetric analysis of the upper airways, was composed of 298 CBCT scans-144 males (18 to 64 years old, mean age $32.04 \pm 12.48$ years), and 154 females (18 to 76 years old, mean age $30.87 \pm 11.47$ years). Due to limitations that hampered the volumetric evaluation of the maxillary sinuses (e.g., mucous disease), only 212 of the 298 CBCT scans were used for this analysis-104 males (18 to 64 years old, mean age $32.40 \pm 12.46$ years), and 108 females (18 to 76 years old, mean age $29.62 \pm 11.44$ years).

All CBCT images were acquired with an i-CAT ${ }^{\circledR}$ Next Generation device (Imaging Sciences International, Hatfield, $\mathrm{Pa}$ ), with the following acquisition parameters: $5 \mathrm{~mA}, 120 \mathrm{kVp}, 17.3 \mathrm{~s}$ scanning time, $0.3 \mathrm{~mm}^{3}$ voxel size, and an extended field of view (FOV) $-23 \times 17 \mathrm{~cm}$. The images were exported in digital imaging and communications in medicine files (DICOM) format and selected in XoranCat ${ }^{\circledR}$ Software version 3.1.62.

\section{Sample classification}

Initially, each patient was classified in regard to skeletal patterns-skeletal malocclusion (Class I, II, or III), and facial type (dolichocephalic, brachycephalic, or mesocephalic), and breathing pattern (nasal breathing, or mouth breathing). To do so, two previously calibrated examiners assessed in consensus the multiplanar reconstructions obtained from the CBCT images, using the Carestream Dental 3D Imaging software (version 3.10.9.0, Atlanta, Georgia, USA).

Skeletal malocclusion was established based on Steiner's cephalometric standards for the SNA, SNB, and ANB angles. The ANB angle was obtained by subtracting the $\mathrm{SNB}$ from the SNA values $(\mathrm{ANB}=\mathrm{SNA}-\mathrm{SNB})$. ANB values 0 to $4=$ skeletal Class I; $A N B>4=$ Class II; and $\mathrm{ANB}<0=$ Class III (Fig. 1). [11, 12] Facial type was determined based on the Vert index, [13] which was obtained from the arithmetic average of five cephalometric measures: facial depth (Po-Or/N-Pog), facial axis angle (N-Ba/ $\mathrm{Pt}-\mathrm{Gn}$ ), lower facial height (Xi-ANS/Xi-Pm), mandibular plane angle (Go-Me/Po-Or), and mandibular arch (Dc$\mathrm{Xi} / \mathrm{Xi}-\mathrm{Pm})$. Resultant values greater than 0.5 determined the brachycephalic type; values lower than -0.5 determined the dolichocephalic type; and values between -0.5 and +0.5 represented the mesocephalic type (Fig. 2). Table 1 shows the definitions of the variables. 


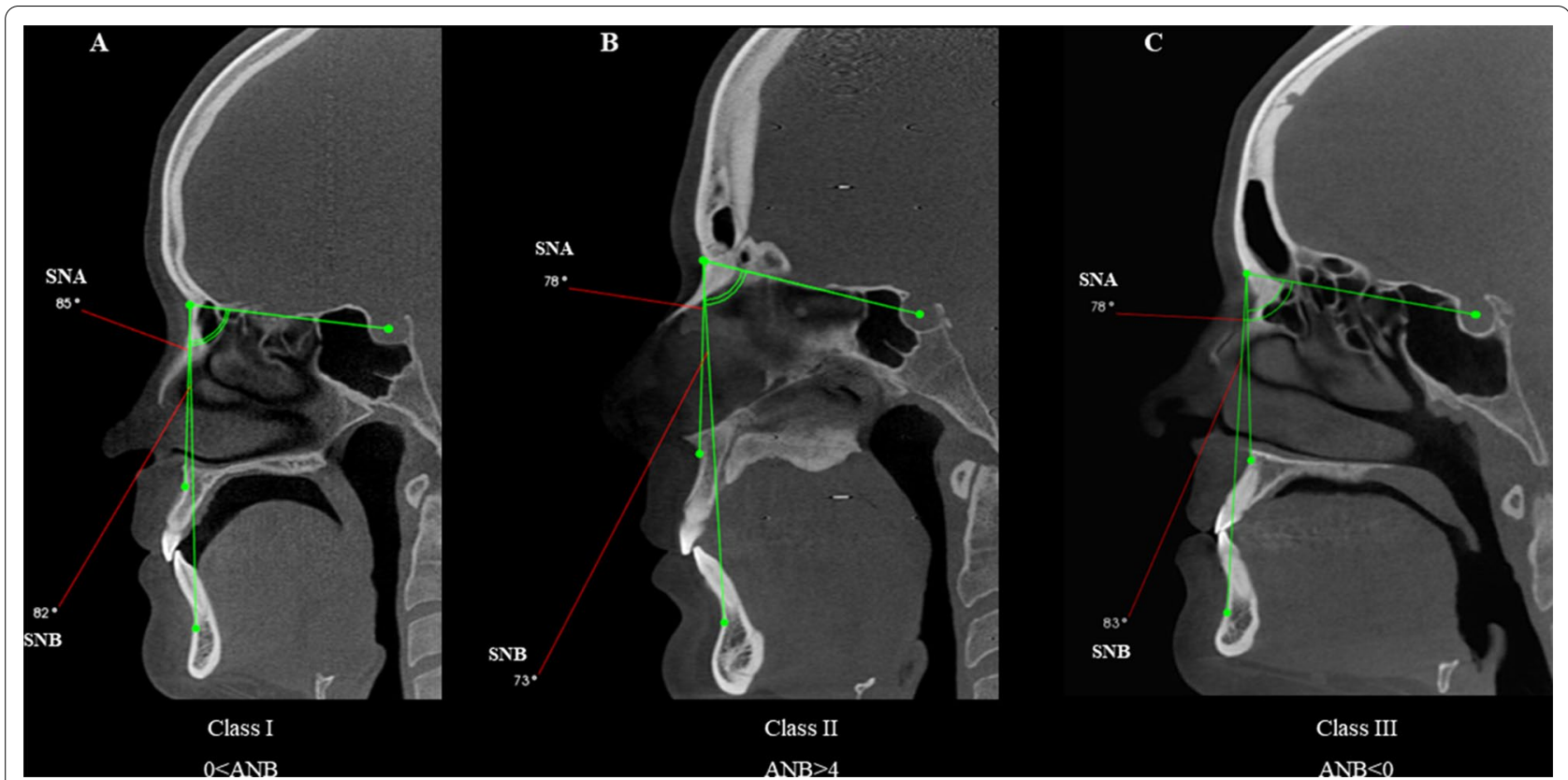

Fig. 1 Sagittal reconstructions of cone-beam computed tomography demonstrating patients' classification according to skeletal malocclusion, based on Steiner's cephalometric standards for the SNA, SNB, and ANB angles. SNB-SNA ANB. A Class I: $0<$ ANB $<4$, B Class II: ANB >4, C Class III: $\mathrm{ANB}<0$

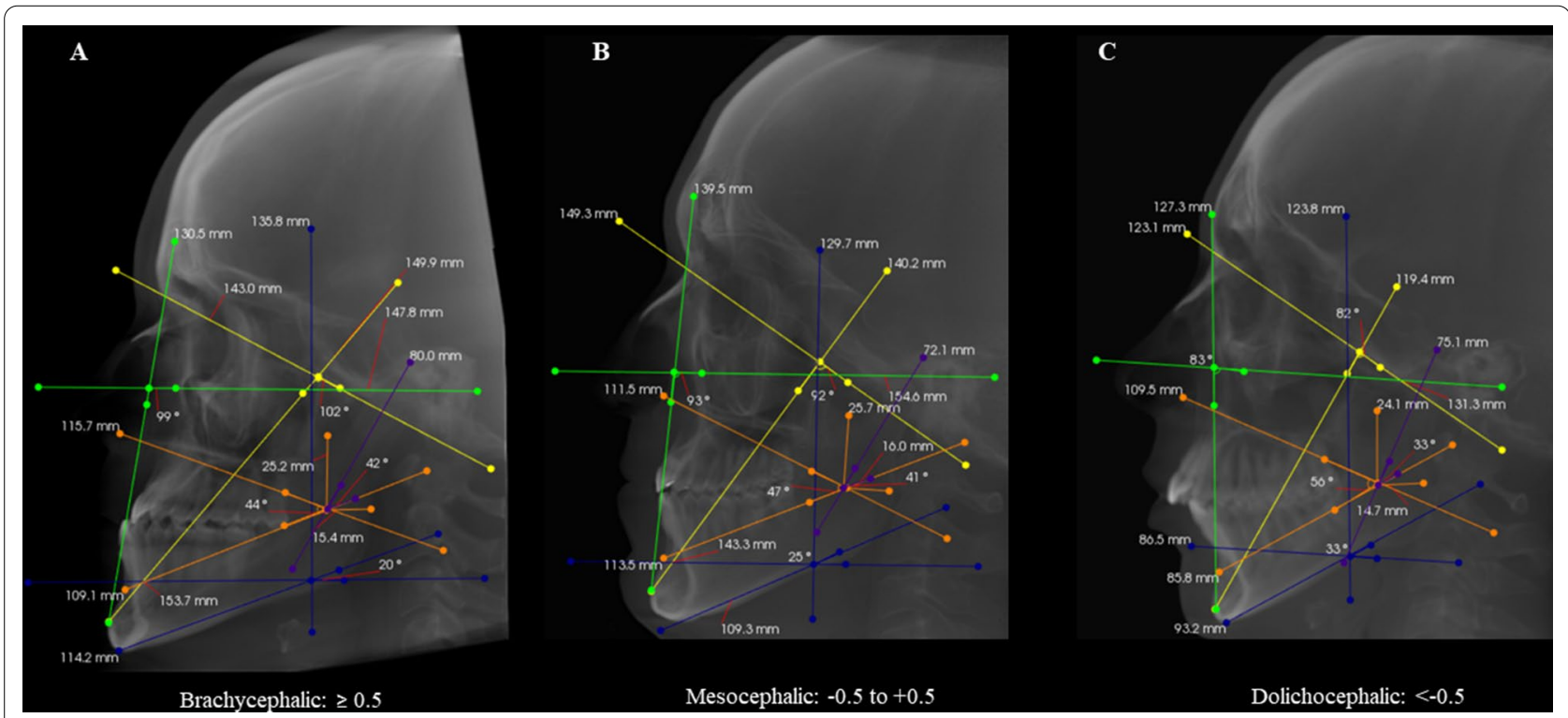

Fig. 2 Sagittal reconstructions of cone-beam computed tomography demonstrating patients' classification according to facial type, based on Vert index. Representative colors of cephalometric measurements: yellow: facial axis; green: facial depth; blue: mandibular plane; orange: lower facial height; and purple: mandibular arch. A Brachycephalic: $\geq 0.5$, B Mesocephalic: -0.5 to +0.5, C Dolichocephalic: $<-0.5$

Regarding the breathing pattern, the classification was based on the hyoid triangle methodology, which considers the position of the hyoid bone (Fig. 3). [1416] Firstly, each CBCT scan was spatially re-oriented so the software's vertical reference line was placed in the median sagittal plane, in the coronal view; then, the horizontal reference line in the sagittal reconstruction, and the vertical reference line in the axial reconstruction were positioned passing through the anterior and posterior nasal spines for standardization [14]. Thus, 
Table 1 Definitions of variables in the classification of individuals regarding skeletal patterns (skeletal malocclusions and facial types)

\begin{tabular}{lll}
\hline Skeletal pattern & Variables & Definition \\
\hline Skeletal malocclusion & SNA & Angle formed from the points Sella (S), Nasion (N) and A \\
& SNB & Angle formed from the points Sella (S), Nasion (N) and B \\
Facial type & Angle formed from the points A, Nasion (N) and B \\
& Facial depth & Angle formed by intersecting the lines Po (Porion)—Or (Orbitale) and N (Nasion)—Pog (Pogonion) \\
& Facial axis angle & Angle formed by intersecting the lines N (Nasion)—Ba (Basion) and Pt (Pterygoid Point)—Gn \\
& (Gnathion) & \\
& Lower facial height & Angle formed by intersecting the lines Xi (Geometric center of the ramus)—ANS (Anterior nasal \\
& spine) and Xi (Geometric center of the ramus)—Pm (Suprapogonion) \\
& Mandibular plane angle & Angle formed by intersecting the lines Go (Gonion)—Me (Menton) and Po (Porion)—Or (Orbitale) \\
& Angle formed by intersecting the lines Dc (Point in the center of the mandibular condyle)—Xi \\
& (Geometric center of the ramus) and Xi (Geometric center of the ramus)—Pm (Suprapogonion) \\
\hline
\end{tabular}

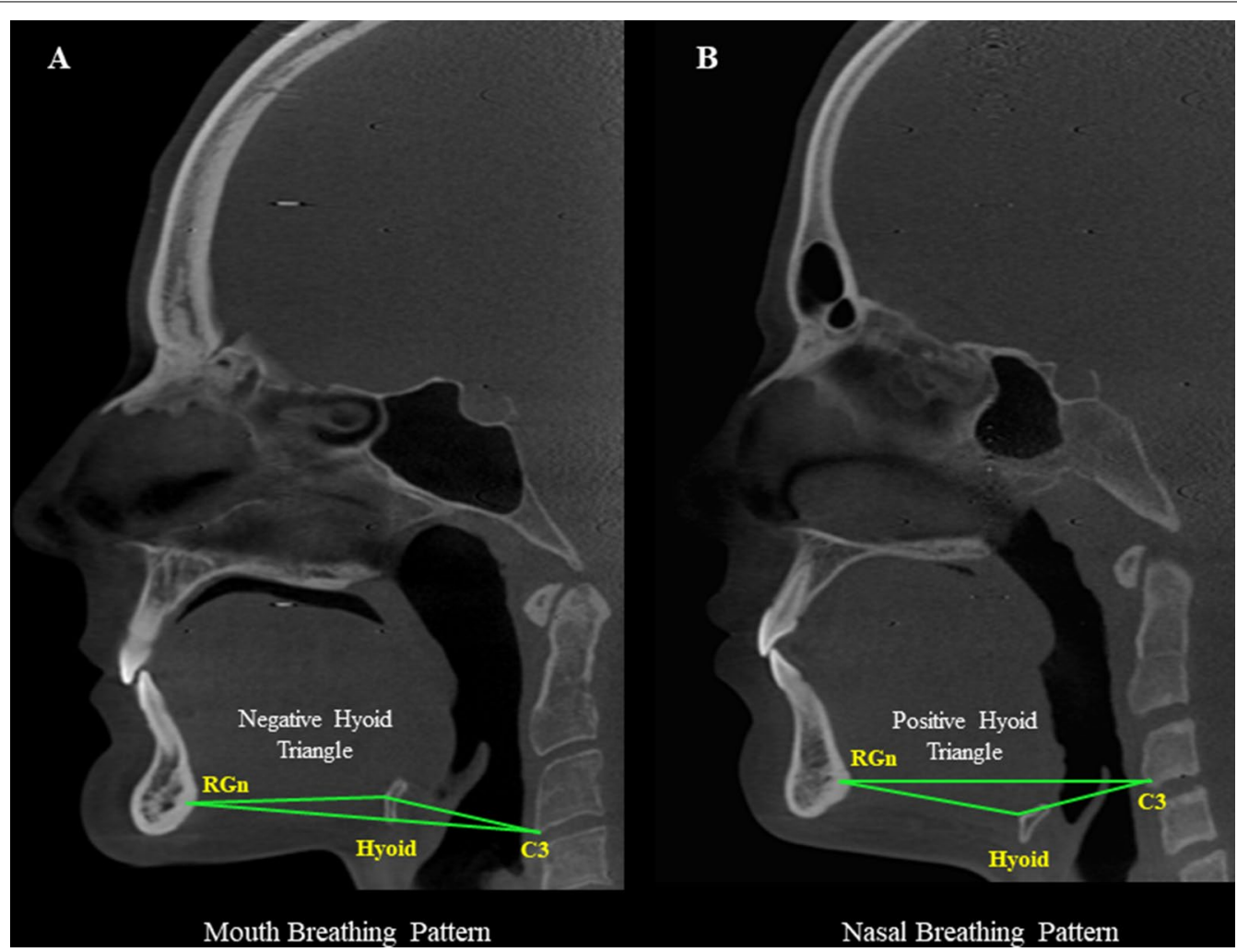

Fig. 3 Classification of patients by breathing pattern based on the hyoid triangle. A Mouth Breathing Pattern, B Nasal Breathing Pattern

in the sagittal view, the hyoid bone was clearly visualized. A line between the most inferior-anterior point of the third cervical vertebra $\left(\mathrm{C}_{3}\right)$ and the most posterior point of the mandibular symphysis (retrognathic cephalometric point-RGn) was drawn, establishing the triangle basis. Another line from $\mathrm{C}_{3}$ to the most anterior point of the hyoid bone was drawn, and then to the RGn point, determining the hyoid triangle. Thus, if the hyoid bone was positioned on or above the $\mathrm{RGn}-\mathrm{C}_{3}$ plane, that meant a higher position of the hyoid, determining a negative triangular position and, therefore, a mouth breathing pattern. Conversely, if the hyoid bone was positioned bellow the $R G n-C_{3}$ plane, that meant a lower position of the hyoid, determining a positive triangular position and, therefore, a nasal breathing pattern. 
Sample distribution according to the classifications is shown in Fig. 4.

\section{Three-dimensional assessment}

The Carestream Dental 3D Imaging software (version 3.10.9.0, Atlanta, Georgia, USA) was used for the morphometric analysis of the hard palate, and the ITK-SNAP v.3.0 (Cognitica, Philadelphia, PA) was employed to evaluate the volumes of the upper airways and maxillary sinuses.

Before initiating the evaluations, the examiners were instructed about the assessment of the hard palate, upper airways, and maxillary sinuses, using scans that were not included in the final sample as examples. Two examiners (3 years of experience in the assessment of $\mathrm{CBCT}$ images) evaluated the scans independently. The evaluations were performed in a low-light and silent environment, using a 24.1-in LCD display (Barco MDRC-2124; Barco., Kortrijk, Belgium) with a resolution of $1920 \times 1200$ pixels.

\section{Morphometric analysis of the hard palate}

Initially, each $\mathrm{CBCT}$ scans was spatially reoriented to standardize the assessment. As the literature does not present a study with a methodology for evaluating the upper teeth roots, the reorientation was arbitrarily developed by the authors. The vertical reference line of the software was placed at the median sagittal plane, in the coronal view; then, in the sagittal reconstruction, the horizontal reference line was positioned passing through the lowest point of the first cervical vertebra, with the
Morphometric on posterior region of the hard palate and upper airways volume analysis (298 CBCT)
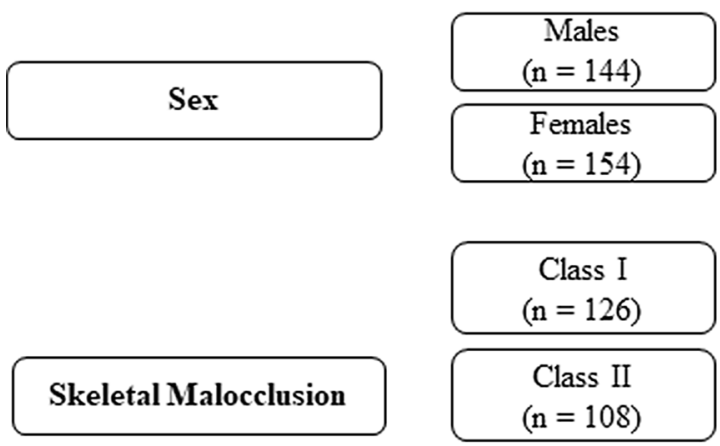

Class III $(\mathrm{n}=64)$
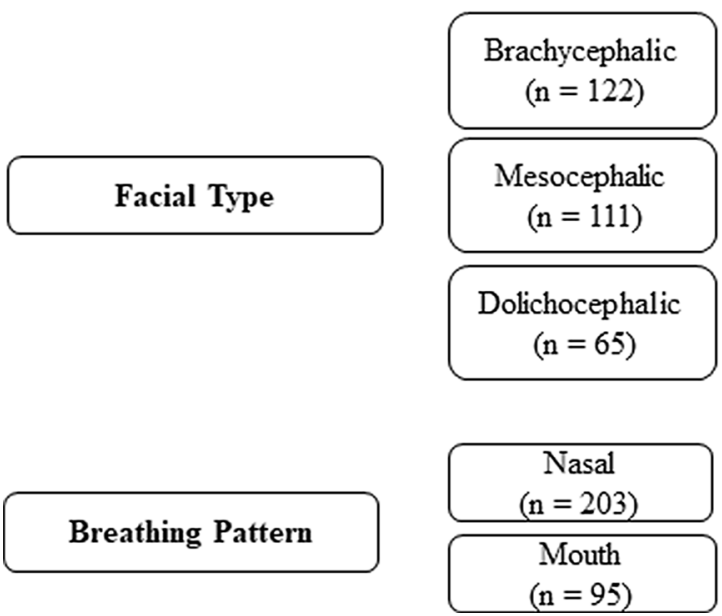

Maxillary sinuses volume analysis (212 CBCT)

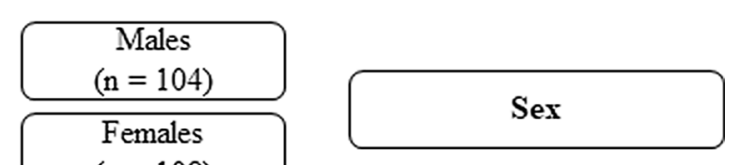

$(n=108)$

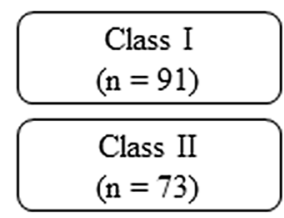

\section{Skeletal Malocclusion}

Class III

$(\mathrm{n}=48)$
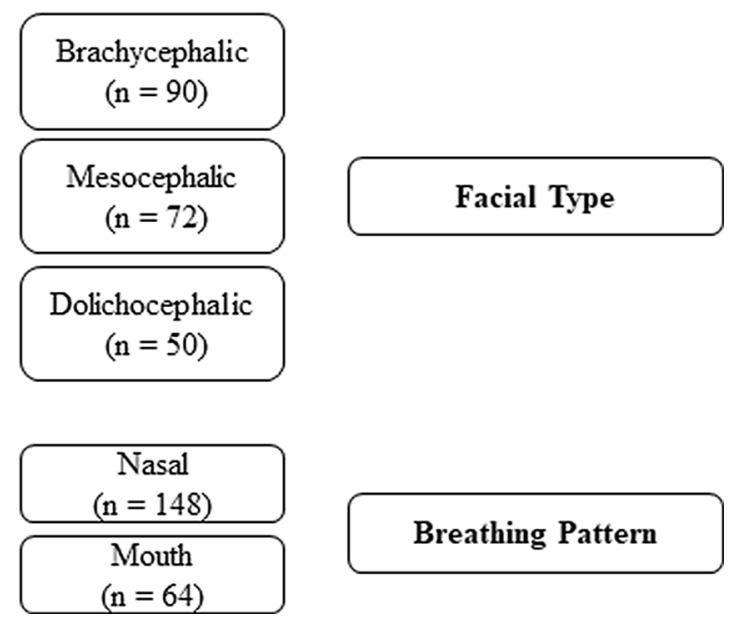

Breathing Pattern

Fig. 4 Diagram of the sample after all classifications have been established 
roots of all teeth being observed in the axial view. Measurements on the posterior region of the hard palate were done at the levels of the upper first premolars, and first molars (Fig. 5).

To perform the measurements of the hard palate at the level of the first premolars, in the axial view, the horizontal reference line of the software was positioned on the palatal roots of the first premolars, crossing the right and left sides of the palate. Then, in the coronal view, with the measuring tool of the software, a horizontal line connecting the palatal bone cortex at the region of the right first premolar to the palatal bone cortex at the region of the left first premolar was drawn, determining the values of posterior width of the hard palate at this level. The same procedure was performed at the first molars level. Furthermore, the height of the hard palate at the levels of the first premolars and first molars was also measured. To do so, a line perpendicular to the horizontal line previously described (posterior width) was established from the most superior point of the hard palate to the center of the horizontal line (Fig. 6).

\section{Volumetric analysis}

The volumetric analysis of the upper airways and maxillary sinuses were performed by semiautomatic segmentation.

In the evaluation of the upper airways, the volumes of the nasopharynx, oropharynx, and of the entire upper airway were analyzed. Firstly, the region of interest (ROI) for segmentation was established according to the following limits: the anterior limit running along the posterior nasal spine, parallel to the sagittal plane; posterior limit passing through the cervical vertebra $\left(\mathrm{C}_{2}\right.$ and $\left.\mathrm{C}_{3}\right)$; lateral limits running along the lateral walls of the pharynx; inferior limit passing tangentially to the medial caudal projection of the third cervical vertebra $\left(C_{3}\right)$, perpendicular to the sagittal plane; and, the superior limit up to the highest point of the nasopharynx [17]. After establishing the ROI, the segmentation was performed by means of three interactive steps: firstly, a threshold range was set to determine the start and end of the segmentation process. A value of -1000 was set for the lower threshold, and a value varying from -660 to -531 was established for the upper threshold, meaning that all voxels with gray values inside that interval were selected to construct the 3D model. After that, "seeds" were placed in the ROI to initiate the segmentation; and lastly, the segmentation evolution was run by selecting its velocity and end. If there was an area that was not defined, a manual adjustment was made by the evaluator. To obtain the volumes of the nasopharynx and oropharynx separately, the software's scalpel tool was used. An oblique cut line was traced over the reference structures: the lower aspect of the first cervical vertebra $\left(C_{1}\right)$, and the posterior nasal spine. The upper airway's total volume, and the volumes of the naso and oropharynx regions were calculated in cubic millimeters $\left(\mathrm{mm}^{3}\right)$ by the software (Fig. 7).

Regarding the maxillary sinuses, the volumes of the left and right sinuses were evaluated, and their total volume was obtained. The examiner established the region of interest (ROI) in the CBCT multiplanar reconstructions, using the lateral, medial, superior, inferior, anterior, and

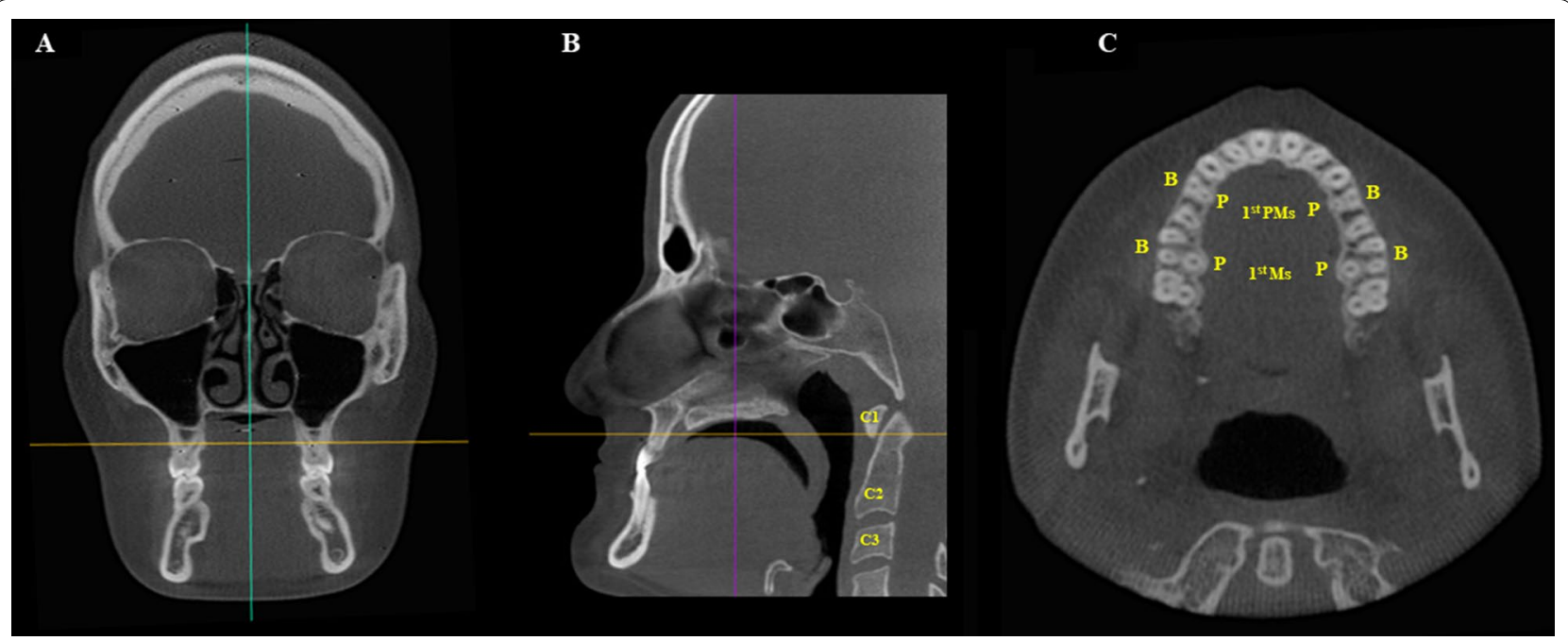

Fig. 5 Coronal section orientation in the morphometric evaluation of the hard palate. A Coronal reconstruction—green line positioned at the median sagittal plane and perpendicular to the yellow line, which is placed over the teeth of the maxilla, $\mathbf{B}$ Sagittal reconstruction-Yellow line passing through the lowest point of the first cervical vertebra $\left(C_{1}\right)$; C Axial reconstruction-maxillary arch showing the individualized roots of the multiradicular teeth. $C_{2}$ : second cervical vertebra; $C_{3}$ : third cervical vertebra; $B$ : buccal; $P$ : palatine; 1 st PMs: first premolars; 1 st Ms: first molars 


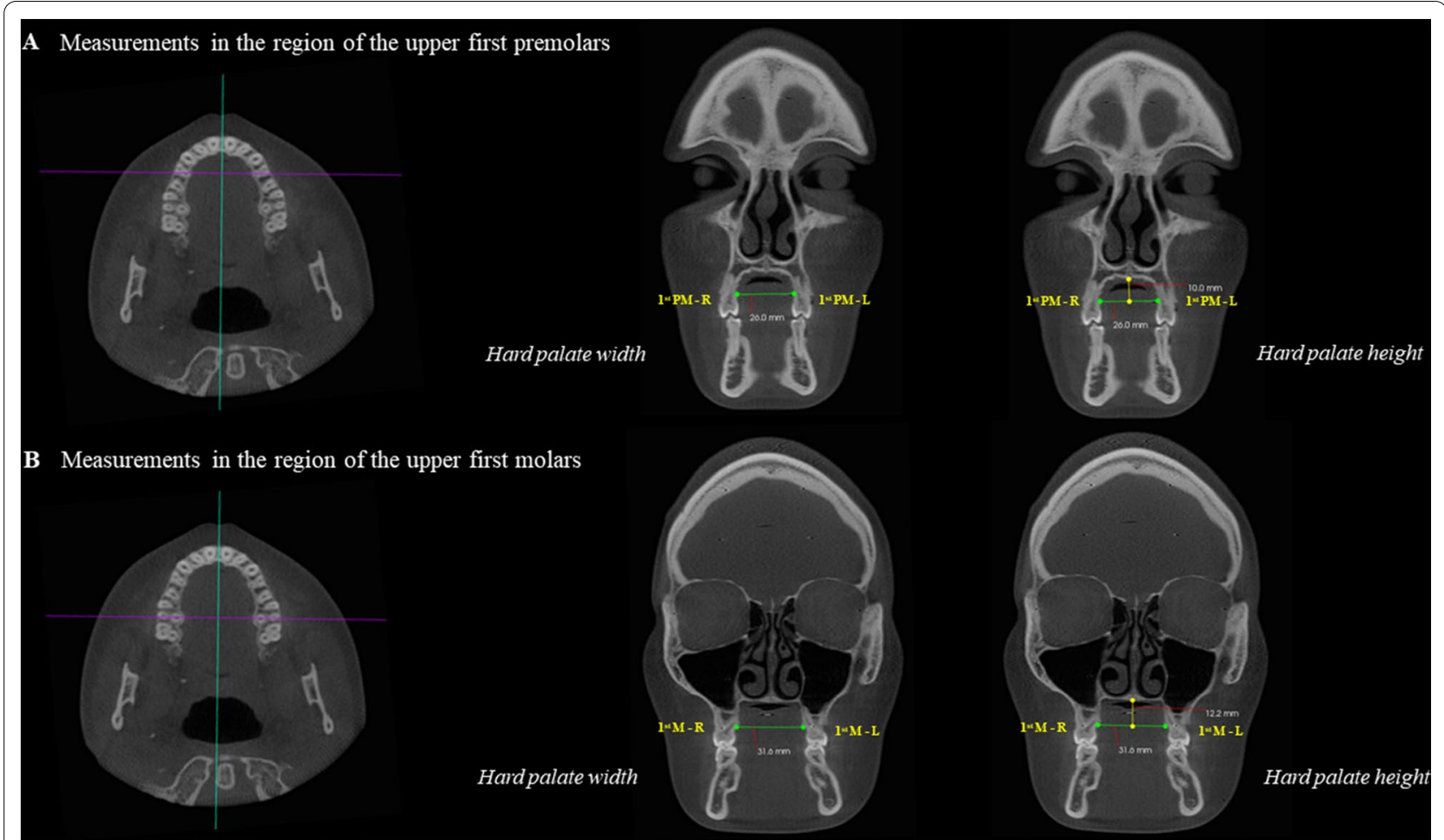

Fig. 6 Morphometric analysis of the hard palate. A and B Axial reconstructions - green line positioned in the median sagittal plane and perpendicular to the purple line, which passes over the palatine roots of the upper first premolars and first molars. A Coronal reconstructionmeasurement of hard palate width (green line) and height (yellow line) at the first premolars region. B Coronal reconstruction-measurement of the hard palate width (green line) and height (yellow line) at the first molars region. $1^{\text {st }}$ PM first premolar; 1st Ms: first molar; R: right; L: left.

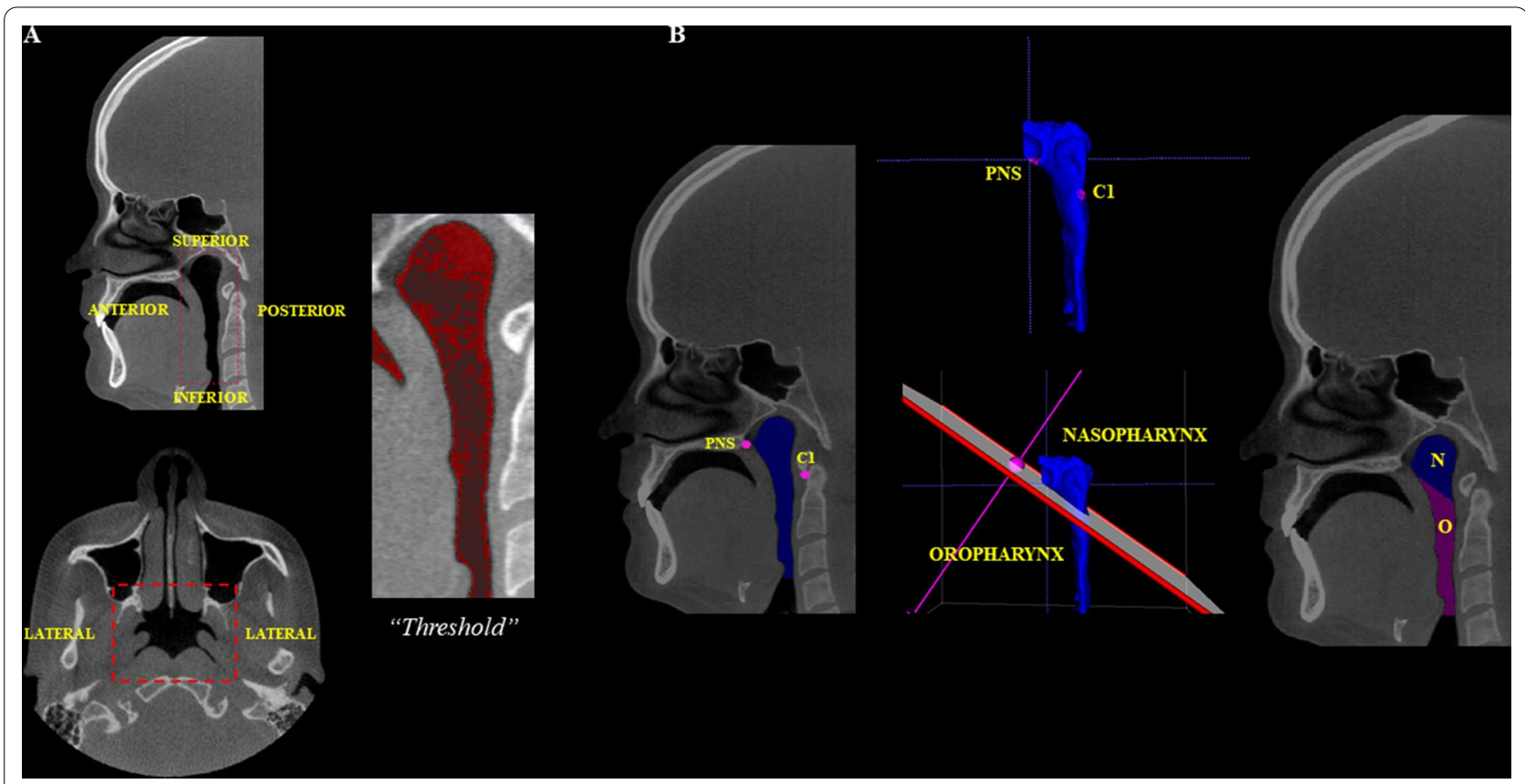

Fig. 7 Volumetric analysis of the upper airways. A Sagittal and axial reconstructions—Region of Interest (ROI) for upper airway segmentation, and threshold selection for semi-automatic airway filling. B Sagittal reconstructions-Upper airways filled, and segmentation for individualization of the nasopharynx (N) — blue region, and oropharynx (O) — pink region. PNS: posterior nasal spine; C1: first cervical vertebra 
posterior walls of the maxillary sinus as limits. [18] Then, the threshold range (lower threshold: - 1000; upper threshold: -678 to -518 for the right sinus, and -710 to -572 for left sinus) was set, and semiautomatic filling of the selected region was performed after placing the "seeds" along the ROI. A manual adjustment was made if there was an area that was not defined. The total volume of the maxillary sinuses was established in $\mathrm{mm}^{3}$ (Fig. 8).

Thirty days after the end of evaluations, part of the sample (30\%) was reassessed to obtain the intraexaminer agreement.

\section{Data analysis}

Intra- and interexaminer agreements were calculated by the intra-class correlation coefficient (ICC) test $(>0.75-$ excellent; 0.40-0.75-moderate, and $<0.40-$ poor). [19].

The normality of the data was verified using the Kolmogorov-Smirnov test. The influence of the studied factors (sex, skeletal malocclusion, facial type, and breathing pattern) on the linear measurements of the hard palate in the regions of the first premolars and first molars was tested by the multi-way analysis of variance (ANOVA).

In order to observe the relationship between the linear measurements of the hard palate and the volumes of the upper airways and maxillary sinuses, multiple linear regression was used. To do so, each factor (width and height of the hard palate at the levels of the first premolars and first molars) was subjected to a simple linear regression to verify the possible influence of each of them on the studied volumes. Then, factors showing $p$-values lower than $0.20(p<0.20)$ were applied together in a multiple linear regression.

The null hypothesis considered that the dimensions of the hard palate are not influenced by sex, skeletal and breathing patterns. It also considered that there is no relationship between the dimensions of the hard palate and the volumes of the upper airways and maxillary sinuses. All analyses were conducted with the Statistical Package for the Social Sciences v.23.0 (SPSS Inc., Chicago, IL, EUA), and the Bioestat ${ }^{\circledR}$ v.5.3 (Instituto de Desenvolvimento Sustentável Mamirauá, Tefé, AM, Brazil) software, with a significance level established at 5\% $(p<0.05)$.

\section{Results}

ICC results revealed excellent intra- and interexaminer agreements for all assessments. For the maxillary sinuses volume, the values of intra- and interexaminer agreements ranged from 0.97 to 0.99 , and from 0.96 to 0.99 , respectively. For the upper airways volume, the intra- and interexaminer agreements ranged from 0.96 to 0.98 , and from 0.77 to 0.94 , respectively; and for the linear measurements of the hard palate, they ranged from 0.89 to 0.93 , and from 0.78 to 0.86 .

Table 2 shows the linear measurements of the hard palate according to sex, skeletal patterns, and breathing

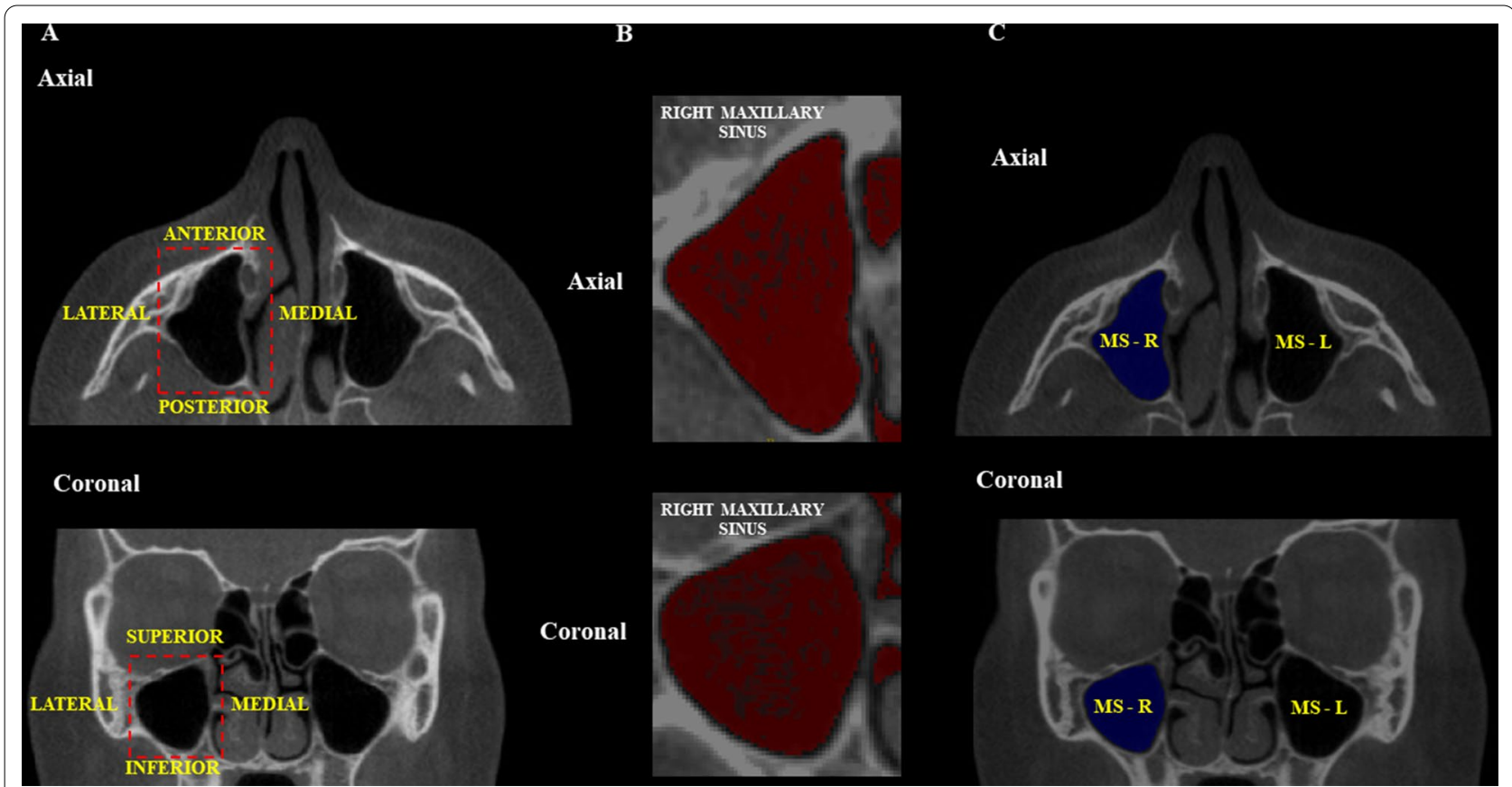

Fig. 8 Volumetric analysis of the maxillary sinuses. A ROI for segmentation of the maxillary sinuses. B Threshold selection for semi-automatic filling of the maxillary sinuses. C Filled maxillary sinus to obtain the volume in $\mathrm{mm}^{3}$. MS: maxillary sinus; $R$ : right; $L$ : left 


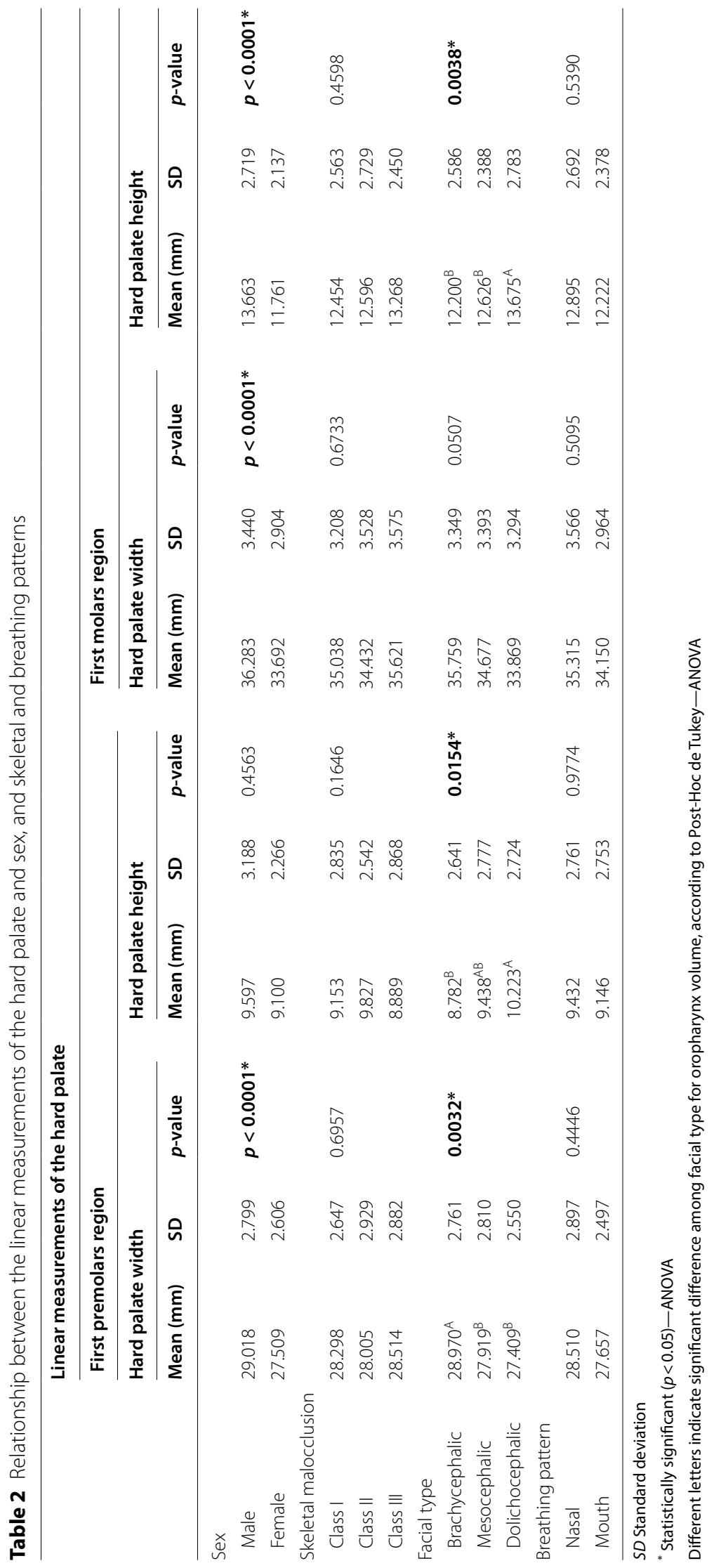


patterns. There was an influence of sex on the hard palate measurements $(p<0.0001)$, except for the height in the first premolars region $(p=0.4563)$. Males presented greater width of the hard palate in both first premolars and first molars regions, and greater values of height of the hard palate in the first molars region. Regarding the facial types, brachycephalic patients had greater values of hard palate width in the first premolars region $(p=0.0032)$ than the other facial types, while dolichocephalic individuals had greater values of hard palate height than brachycephalics $(p=0.0154)$. For the height of the hard palate in the first molars region, dolichocephalic patients had greater values than the other facial types $(p=0.0032)$. Skeletal malocclusion, and breathing pattern did not influence the measurements of the hard palate $(p>0.05)$.

Furthermore, in order to observe the relationship between the measurements of the posterior region of the hard palate (width and height) and the volumes of the upper airways and maxillary sinuses, a multiple linear regression analysis was performed (Table 3). It was found an association between the measurements of the hard palate at the first premolars region and the upper airways volume: width and height of the hard palate and the total volume of the upper airway $(p=0.018$ and 0.038 , respectively); width and height of the hard palate and the nasopharynx volume ( $p=0.013$ and 0.035 , respectively); and width of the hard palate and the oropharynx volume $(p=0.024)$. In this association, the greater the height of the hard palate, the lower the volume of the upper airways, and the greater the width of the hard palate, the higher the volume of the upper airways. It was also observed an association between the width and height of the hard palate at the first molars region and the total volume of the maxillary sinuses $(p<0.0001)$, in which the greater the width and height of the hard palate, the greater the volume of the maxillary sinuses.

\section{Discussion}

The hard palate is one of the central structures of the stomatognathic system, and it presents great anatomical and clinical importance, as it assists in different orofacial functions, including breathing $[1,5]$. When there is an imbalance between the structures of the stomatognathic system due to airway obstruction, or due to changes in the direction of growth and development of the face, the hard palate may change and adapt its morphology, position, and function $[2,3,5]$. In this study, the dimensions of width and height of the posterior region of the hard palate in patients of different sexes, and skeletal and breathing patterns were assessed. It was also investigated if there was an association between the measurements of the hard palate and the volumes of the upper airways and maxillary sinuses. It was found that sex and facial type influence the dimensions of the hard palate, and there was an association between the measurements of the

Table 3 Multiple linear regression for the association between the volumes of the upper airways and maxillary sinuses and the linear measurements of the hard palate

\begin{tabular}{|c|c|c|c|c|c|}
\hline Multiple linear regression & Factors & BC & SE & $\mathrm{Cl}(95 \%$ for B) & $p$-value \\
\hline \multicolumn{6}{|l|}{ Upper airways } \\
\hline \multirow[t]{3}{*}{ Total volume } & HP width (1st PM) & 0.189 & 0.197 & $(0.082-0.857)$ & $0.018^{*}$ \\
\hline & HP height (1st PM) & -0.121 & 0.147 & $(-0.595--0.017)$ & $0.038^{*}$ \\
\hline & HP width (1st M) & -0.042 & 0.159 & $(-0.398-0.228)$ & 0.593 \\
\hline \multirow[t]{3}{*}{ Nasopharynx volume } & HP width (1st PM) & 0.198 & 0.083 & $(0.045-0.373)$ & $0.013^{*}$ \\
\hline & HP height (1st PM) & -0.123 & 0.062 & $(-0.255--0.009)$ & $0.035^{*}$ \\
\hline & HP width (1st M) & -0.033 & 0.068 & $(-0.162-0.104)$ & 0.669 \\
\hline \multirow[t]{2}{*}{ Oropharynx volume } & HP width (1st PM) & 0.132 & 0.105 & $(0.032-0.446)$ & $0.024^{*}$ \\
\hline & HP height (1st PM) & -0.096 & 0.107 & $(-0.386-0.034)$ & 0.100 \\
\hline \multicolumn{6}{|l|}{ Maxillary sinuses } \\
\hline \multirow[t]{2}{*}{ Total volume } & HP width (1st M) & 0.291 & 0.173 & $(0.486-1.169)$ & $p<0.0001 *$ \\
\hline & HP height $\left(1^{\text {st }} \mathrm{M}\right)$ & 0.358 & 0.229 & $(0.895-1.798)$ & $p<0.0001 *$ \\
\hline
\end{tabular}

HP Hard palate

1st PM Region of the first premolars

1st $M$ Region of the first molars

$B C$ Beta coefficient

SE Standard error

Cl 95\% Confidence interval for beta

${ }^{*}$ Significant association $(p<0.05)$, according to Multiple linear regression 
hard palate and the volumes of the maxillary sinuses and upper airways.

About the morphometric analysis of the hard palate, significant differences were found between sexes, and among the facial types, for some measurements. Males had greater width of the hard palate at the first molars region, and greater values of height of the hard palate at the first premolars region, corroborating previous studies $[20,21]$. For facial types, in general, dolichocephalic individuals presented greater height of the hard palate in the first premolars and molars regions, whereas brachycephalic patients had greater width of the hard palate in the first premolars region. In view of this result, it is possible to observe the influence of the vertical growth trend in the shape of the hard palate, since the greater vertical tendency of growth (dolichocephalic) presented greater height of the hard palate, while the greater horizontal tendency (brachycephalic) showed greater width of the hard palate. These results corroborate the theories presented by Vucic et al. [2] and Miranda-Viana et al. [3] that the bone structures of the craniofacial complex adapt to variations in craniofacial growth trends. Conversely, two previous studies reported no significant differences among facial types for linear measurements of the hard palate [22, 23]. However, these studies were performed on plaster models, which present deformations inherent to molding and plastering, while in our study the hard palate was evaluated by means of CBCT scans, which provide three-dimensional images with no magnification or distortion. No significant differences were identified among the different skeletal malocclusions for the hard palate morphometry. This may be related to the fact that the skeletal malocclusions are defined by the position of the mandible in relation to the base of the skull, not influencing the width and/or height of the hard palate. To our knowledge, no other study in the literature has performed this type of analysis. Therefore, further studies are encouraged in order to confirm or refute our hypothesis. In regard to the breathing pattern, no significant difference was observed between mouth and nasal breathing for the morphometric analysis of the hard palate, which is in disagreement with prior researches $[6,7,24]$. These different findings are believed to have occurred because these studies were carried out using plaster models. Another factor is that, differently from our sample of adult patients, these studies were carried out in children, in which the bone and integumentary tissues are not yet fully developed. Thus, we believe that the body may adapt to the breathing pattern after the individual is fully grown.

A linear regression was developed to investigate a possible relationship between the hard palate measurements and the volumes of the upper airways and maxillary sinuses. It was found an association between the width and height of the hard palate at the level of the first premolars and the volumes of the upper airways (total volume) and nasopharynx; and between the hard palate width and the volume of the oropharynx. According to our results, the greater the height of the hard palate, the lower the volume of the upper airways; and the greater the width of the hard palate, the higher the volume of the upper airways. It was also found an association between the width and height of the hard palate at the level of the first molars and the volume of the maxillary sinuses, in which the greater the width and height of the hard palate, the greater the volume of the maxillary sinuses. Grauer et al. [25] and Gupta et al. [26] reported an association between the facial width (distance from right to left zygomatic bone) and the upper airway volume. Their results seem to corroborate those of the present study, in which the width of the hard palate showed to be related with the volume of the upper airways, since, although we have measured different bones, both studies assessed the horizontal dimensions of craniofacial structures. Regarding the association between the hard palate and the volume of the maxillary sinuses, this is the first work to perform this analysis. Thus, it is not possible to compare our results with the literature. Therefore, future studies evaluating this association are encouraged, since the results reported here may contribute to additional clinical information, and to assist in the elaboration of the treatment planning in different areas, such as orthodontics, surgery, and otorhinolaryngology.

The authors consider that the relationship between the linear measurements of the hard palate at the first premolars region and the volume of the upper airways may be due to the craniofacial growth trend. Dolichocephalic patients have a tendency of vertical craniofacial growth, presenting a narrower and deeper hard palate. On the other hand, brachycephalic patients have a tendency of horizontal craniofacial growth, presenting a regular and larger hard palate [3, 22, 24]. This is in concordance with the results of this study, in which dolichocephalic and brachycephalic individuals presented greater values of height and width of the hard palate, respectively, at this region. In addition, the study of Fernandes et al. [27] observed a trend towards vertical growth when the oropharynx permeability is reduced. In our research, dolichocephalic patients presented lower volume of the airways at the oropharynx region than brachycephalics. Given these characteristics, there is an indication that the width and height of the posterior region of the hard palate are associated with the volume of the upper airways, as patients with greater height and width of the hard palate presented lower and greater values of volume, respectively. 
One may consider that the cross-sectional area of some segments of the upper airways, especially their narrower part, may influence the resistance to the airflow more significantly than their volume. In the present study, we opted to evaluate the volume of the airways instead of measuring cross-sectional areas, since we did not aim at evaluating if there is a relationship between the hard palate dimensions and a possible increased resistance to the airflow. However, one of the findings in this study was the association between the width of the hard palate and the volume of the oropharynx, in which the lower the width of the hard palate, the lower the volume of the oropharynx. Because previous studies in the literature $[28,29]$ have reported that the oropharynx has the narrower area of the upper airways, it is possible that there is a relationship between the width of the hard palate and the crosssectional area of the oropharynx as well, i.e., a lower width of the hard palate may be related to an increased resistance to the airflow. However, further studies are necessary to confirm or refute this hypothesis.

In view of the presented and discussed results, it is important to highlight that the sample size of this study was representative of the assessed population, and it was homogeneously distributed within each variable evaluated. Thus, it is considered that individual variations did not affect the study's results and conclusions. In addition, we understand that the association of the linear dimensions (width and height) of the posterior region of the hard palate with the volumes of the upper airways and maxillary sinuses shows the clinical and anatomical importance of this bone, which may provide clinical information for procedures involving the airways and/or the maxillary sinuses.

Therefore, future studies investigating this clinical relationship in the areas of oral and maxillofacial surgery, orthodontics and otorhinolaryngology are encouraged.

\section{Conclusion}

The width and height of the hard palate are influenced by sex and facial type, but not by skeletal malocclusion or breathing pattern. Also, there is an association between these dimensions and the volumes of the upper airways and maxillary sinuses. Thus, professionals should be aware of possible changes in the upper airways and/or maxillary sinuses volumes when surgical procedures are performed in the maxillary region.

\footnotetext{
Abbreviations

3D: Tridimensional; ANOVA: Analysis of variance; $C_{1}$ : First cervical vertebra; $\mathrm{C}_{2}$ : Second cervical vertebra; $\mathrm{C}_{3}$ : Third cervical vertebra; CBCT: Cone beam computed tomography; FOV: Field of View; IRB: Institutional review board; LCD: Liquid crystal display; mm: Millimeters; RGn: Retrognathic cephalometric point; ROI: Region of interest.
}

\section{Acknowledgements}

This study was financed in part by the Coordenação de Aperfeiçoamento de Pessoal de Nível Superior_Brasil (CAPES)_Finance Code 001. The authors gratefully acknowledge financial support from CAPES-Brazil, Coordenação de Aperfeiçoamento de Pessoa de Ensino Superior, at UNICAMP. The authors deny any conflicts of interest related to this study.

\section{Authors' contributions}

All authors have read and approved the manuscript. MV-Conceptualization, investigation, writing and revising the draft critically for important intellectual content, final approval of the version to be published and agreement to be accountable for all aspects of the work. DF-Conceptualization, investigation, formal analysis, and interpretation of data for the work, writing and revising the draft critically for important intellectual content and agreement to be accountable for all aspects of the work. AM-Revising the draft critically for important intellectual content and agreement to be accountable for all aspects of the work. AG-Revising the draft critically for important intellectual content, final approval of the version to be published and agreement to be accountable for all aspects of the work. YN-Supervisor; Conceptualization, investigation, revising the draft critically for important intellectual content, final approval of the version to be published and agreement to be accountable for all aspects of the work.

\section{Funding}

This study was financed in part by the Coordenação de Aperfeiçoamento de Pessoal de Nível Superior-Brasil (CAPES) in the role design of the study and collection, analysis, and interpretation of data-Finance Code 001.

\section{Availability of data and materials}

The datasets used and/or analyzed during the current study are available from the corresponding author on reasonable request.

\section{Declarations}

\section{Ethics approval and consent to participate}

This study was carried out after local Institutional Review Board (Ethics Committee for Human Research of the Piracicaba Dental School) approval under the protocol number \#3.491.476. All methods were performed according to the relevant guidelines and regulations of the Declaration of Helsinki. All the research participants signed the informed consent form, and the use of the material was obtained for this study.

\section{Consent for publication}

Not applicable.

\section{Competing interests}

The authors declare that they have no competing interests.

\section{Author details}

${ }^{1}$ Department of Oral Diagnosis - Oral Radiology Area, Piracicaba Dental School, University of Campinas, Piracicaba, SP, Brazil. ${ }^{2}$ Oral Radiology Area, Dental School, Federal University of Mato Grosso Do Sul, Campo Grande, MS, Brazil.

Received: 10 May 2021 Accepted: 14 July 2021

Published online: 20 July 2021

\section{References}

1. Janssen R, Moisik SR, Dediu D. Modelling human hard palate shape with Bézier curves. PLoS ONE. 2018;13:1-38. https://doi.org/10.1016/j.ajodo. 2018.09.014.

2. Vucic S, Dhamo B, Jaddoe VWV, Wolvius EB, Ongkosuwito EM. Dental development and craniofacial morphology in school-age children. Am J Orthod Dentofacial Orthop. 2019;156:229-37. https://doi.org/10.1016/j. ajodo.2018.09.014.

3. Miranda-Viana M, Freitas DQ, Gomes AF, Prado FB, Nejaim Y. Classification and morphological analysis of the hard palate in cone-beam computed 
tomography scans: a retrospective study. J Oral Maxillofac Surg 2021;79:1-13. https://doi.org/10.1016/j.joms.2020.11.024.

4. Pereira TS, de Oliveira FCM. Association between harmful oral habits and the structures and functions of the stomatognathic system: perception of parents/guardians. CoDAS. 2017;29:1-6. https://doi.org/10.1590/23171782/20172015301.

5. Thilander B. Basic mechanisms in craniofacial growth. Acta Odontol Scand. 1995;53:144-51. https://doi.org/10.3109/0001635950900596.

6. Tang $\mathrm{H}$, Liu Q, Lin JH, Zeng H. Three-dimensional morphological analysis of the palate of mouth-breathing children in mixed dentition. Hua Xi Kou Qiang Yi Xue Za Zhi. 2019;37:389-93. https://doi.org/10.7518/hxkq.2019. 04.009.

7. Berwig LC, Silva AM, Côrrea EC, Moraes AB, Montenegro MM, Ritzel RA. Hard palate dimensions in nasal and mouth breathers from different etiologies. J Soc Bras Fonoaudiol. 2011;23:308-14. https://doi.org/10.1590/ s2179-64912011000400004.

8. Lione R, Buongiorno M, Franchi L, Cozza P. Evaluation of maxillary arch dimensions and palatal morphology in mouth-breathing children by using digital dental casts. Int J Pediatr Otorhinolaryngol. 2014;78:91-5. https://doi.org/10.1016/.i.jporl.2013.09.028.

9. Agacayak KS, Gulsun B, Koparal M, Atalay YAO, et al. Alterations in maxillary sinus volume among oral and Nasal Breathers. Med Sci Monit. 2015;21:18-26. https://doi.org/10.12659/MSM.891371.

10. El Nahass H, Naiem SN. Palatal bone dimensions on cone beam computed tomography. Implications for the palate as autogenous donor site: an observational study. Int J Oral Maxillofac Surg. 2016;45:99-103. https:// doi.org/10.1016/j.ijom.2015.09.006.

11. Steiner CC. Cephalometrics for you and me. Am J Orthod. 1953;39:72955. https://doi.org/10.1016/0002-9416(53)90082-7.

12. Plaza SP, Reimpell A, Silva J, Montoya D. Relationship between skeletal Class II and Class III malocclusions with vertical skeletal pattern. Dental Press J Orthod. 2019;24:63-72. https://doi.org/10.1590/2177-6709.24.4. 063072.oar.

13. Costa ED, Peyneau PD, Bolzan GP, Nejaim Y, Caruso JM, Roque-Torres GD. Guideline for manual classification of the facial profiles by CBCT image. Rev Odontol Bras Central. 2021;30:154-61. https://doi.org/10.36065/ robrac.v30i89.149.

14. Da Costa ED, Roque-Torres GD, Brasil DM, Bóscolo FN, de Almeida SMAG. Correlation between the position of hyoid bone and subregions of the pharyngeal airway space in lateral cephalometry and cone beam computed tomography. Angle Orthod. 2017;87:688-95. https://doi.org/ 10.2319/022217-133.1.

15. Chaves TC, de Andrade e Silva TS, Monteiro SA, Watanabe PC, Oliveira ASGD (2010) Craniocervical posture and hyoid bone position in children with mild and moderate asthma and mouth breathing. Int J Pediatr Otorhinolaryngol. 74:1021-7. Doi: https://doi.org/10.1016/j.jporl.2010.05. 031

16. Chung Leng Muñoz I, Beltri Orta P. Comparison of cephalometric patterns in mouth breathing and nose breathing children. Int J Pediatr Otorhinolaryngol. 78:1167-72, 2014. http://doi.org/https://doi.org/10.1016/j. ijporl.2014.04.046

17. Brasil DM, Kurita LM, Groppo FC, Haiter-Neto F. Relationship of craniofacial morphology in 3-dimensional analysis of the pharynx. Am J Orthod
Dentofac Orthop. 2016;149:683-91. https://doi.org/10.1016/j.ajodo.2015. 11.021.

18. Farias Gomes A, de Oliveira GT, Yamasaki MC, Groppo FC, Haiter Neto F, Possobon RF. Development and validation of a formula based on maxillary sinus measurements as a tool for sex estimation: a cone beam computed tomography study. Int J Legal Med. 2019;133:1241-9. https:// doi.org/10.1007/s00414-018-1869-6.

19. Fleiss JL, Cohen J. The equivalence of weighted kappa and the intraclass correlation coefficient as measures of reliability. Educ Psychol Meas. 1973;33:613-9. https://doi.org/10.1177/001316447303300309.

20. Mustafa AG, Tashtoush AA, Alshboul OA, Allouh MZ, Altarifi AA. Morphometric Study of the Hard Palate and Its Relevance to Dental and Forensic Sciences. Int J Dent. 2019;1-6:2019. https://doi.org/10.1155/2019/16873 45.

21. Berwig LC, Marquezan M, Milanesi JM, Montenegro MM, Ardenghi TM, Toniolo da Silva AM. Do gender and age influence hard palate dimensions? A systematic review. Codas. 30:1-14, 2018. http://doi.org/https:// doi.org/10.1590/2317-1782/20182017216

22. Esteves A, Bommarito S. Evaluation of palatine depth and dimensions of the upper dental arch in patients with malocclusion and different facial types. Rev Dent Press Ortod e Ortop Facial. 2007;12:84-98. https://doi. org/10.1590/S1415-54192007000400010.

23. Ahmed HM, Alkhawaja NF, Nahidh M. Assessment of palatal dimensions in a sample of Iraqi adults with different facial forms. Iraqi Orthod J. 2014:10:8-11.

24. Trevisan ME, Bellinaso JH, Pacheco Ade B, Augé LB, Silva AM, Corrêa EC. Respiratory mode, nasal patency and palatine dimensions. Codas. 2015;27:201-6. https://doi.org/10.1590/2317-1782/20152014177.

25. Grauer D, Cevidanes LS, Styner MA, Ackerman JL, Proffit WR. Pharyngeal airway volume and shape from cone-beam computed tomography: relationship to facial morphology. Am J Orthod Dentofacial Orthop. 2009;136:805-14. https://doi.org/10.1016/j.ajodo.2008.01.020.

26. Gupta JV, Makhija PG, Gupta KC. Does a correlation exist between nasal airway volume and craniofacial morphology: a cone beam computed tomography study. Indian J Dent Res. 2016;27:359-63. https://doi.org/10. 4103/0970-9290.191882.

27. Fernandes P, Pinto J, Ustrell-Torrent J. Relationship between oro and nasopharynx permeability and the direction of facial growth. Eur J Paediatr Dent. 2017;18:37-40. https://doi.org/10.23804/ejpd.2017.18.01.08.

28. Nejaim Y, Aps JKM, Groppo FC, Haiter NF. Evaluation of pharyngeal space and its correlation with mandible and hyoid bone in patients with different skeletal classes and facial types. Am J Orthod Dentofacial Orthop. 2018;153:825-33. https://doi.org/10.1016/j.ajodo.2017.09.018.

29. Zheng ZH, Yamaguchi T, Kurihara A, Li HF, Maki K. Three-dimensional evaluation of upper airway in patients with different anteroposterior skeletal patterns. Orthod Craniofac Res. 2014;17:38-48. https://doi.org/10. 1111/ocr.12029.

\section{Publisher's Note}

Springer Nature remains neutral with regard to jurisdictional claims in published maps and institutional affiliations.

Ready to submit your research? Choose BMC and benefit from

- fast, convenient online submission

- thorough peer review by experienced researchers in your field

- rapid publication on acceptance

- support for research data, including large and complex data types

- gold Open Access which fosters wider collaboration and increased citations

- maximum visibility for your research: over 100M website views per year

At BMC, research is always in progress.

Learn more biomedcentral.com/submissions 